scientific interest has been matched in popular media, news magazines, newspaper headlines, television shows, best-selling books, and movies such as Outbreak. (See related Medical News story on emerging infections.)

FROM: Pinner RW, Teutsch SM, Simonsen L, et. al. Trends in infectious disease mortality in the United States. JAMA 1996;275:189-193.

\section{Journals Feature Emerging Infections}

Thirty-six journals in 21 countries agreed to devote all or part of one of their issues to the subject of emerging and reemerging global microbial threats. This international collaboration will publish more than 200 articles. ${ }^{1}$ The first-ever global theme issue of medical journals was coordinated by three editors, Linda Hawes Clever, of the Western Journal of Medicine, Magne Nylenna, MD, editor of the Journal of the Norwegian Medical Association, and George D. Lundberg, MD, editor of the Journal of the American Medical Association (JAMA). Research on tuberculosis, toxigenic Escherichia coli, drug-resistant pneumococcal pneumonia, hepatitis $\mathrm{B}$ and $\mathrm{C}$, cholera, and AIDS will be presented to the readers of journals from Buenos Aires to Syndey to Johannesburg to Beijing. Hantavirus infection is identified in Argentina, Bolivia, and Uruguay, and cat-scratch fever is reviewed in the Netherlands.

Some of the articles offer hope, shedding light on successes and renewed efforts at combating infectious disease. In Iceland, for example, decline in the use of antimicrobials attributable to public education and legislation has been followed by reduced rates of resistant streptococcal pneumonia, and, in the Americas, the Pan American Health Organization has led efforts to eliminate measles from the Western Hemisphere.

An editorial in the January 17,1996 , issue of $J A M A$ by Joshua Lederberg, chair of the Institute of Medicine's Committee on Emerging Infections, notes the importance of this attention to the issue of emerging infections, to reinforce and clarify our consensual medical scientific perspectives, and the "reinforcement they give to the public explicators of contingencies, and [to] government. ${ }^{2}$

"Citations for all articles to be published in the 36 journals are available through the JAMA home page of the American Medical Association's World Wide Web site: http//www.ama-assn.org.

FROM: 1. Winker MA, Flanagin A. Infectious disease: a global approach to a global problem. JAMA 1996;275(3):245-246.

2. Lederberg J. Infection emergent. JAMA 1996;275(3): 243-244.

\section{Terrorist Access to Biologic Agents}

Federal health officials are concerned over a national tissue association's release of a stock culture of Yersinia pestis (plague Bacillus) to a private citizen in Ohio who purported to be a member of the American Society of Microbiology.
In recent testimony given before a US Senate Judiciary Committee, Dr. James Hughes, Director of CDC's National Center for Infectious Disease, testified that "there are a number of federal regulations that address the shipping and handling of infectious agents, however, they are not completely effective at controlling possession and transfer of human infectious agents." Hughes pointed out that, even though there may be up to a few thousand interstate transfers of dangerous human infectious agents for legitimate scientific research, the Ohio incident was the only incident of inappropriate transfer that has ever been reported. Dr. Hughes said, "the goal is to strike a balance between assuring the availability of infectious and recombinant DNA materials to the scientific and medical community for important public health and biotechnical research and preventing access to these agents for other than legitimate scientific and medical purposes.”

Dr. David Satcher, CDC's director, recently sent a letter to the presidents of a number of associations and groups whose members work with pathogenic agents. He pointed out that the CDC was very concerned about the threat of terrorist activity involving the use of biologic agents and the illicit use and interstate transport of certain human pathogenic agents. He requested that organizations and professional microbiologists who authorize the acquisition and transfer of dangerous human infectious agents increase their vigilance to minimize the risk of illicit access to these agents. Requests for agents that cause anthrax, botulism, brucellosis, plague, Q-fever, tularemia, and any agent classified for work at Biosafety Level 4 (ie, Ebola and most other hemorrhagic viruses) should be reviewed to determine if they are being used for legitimate medical or scientific purposes. Suspicious inquiries or transactions should be reported to CDC's Office of Health and Safety (404-639-3235).

CDC co-chairs a federal interdepartmental working group that is developing regulations regarding the acquisition and transfer of certain biologic agents. These regulations will be developed with input from professional organizations, the research community, and law enforcement authorities. A notice of proposed rulemaking will be published in the Federal Register. In addition, the Department of Justice is working to strengthen relevant criminal statutes to enable prosecution of those who attempt to gain illicit access to these biologic agents.

FROM: 1. Satcher D. Letter to professional associations that handle pathogenic agents. March 12, 1996.

2. Testimony by James Hughes, MD, before the US Senate Judiciary Committee, March 6, 1996.

\section{OSHA Revises TB Enforcement}

On February 9, 1996, OSHA issued "Enforcement Procedures and Scheduling for Occupational Exposure to Tuberculosis" (OSHA Instruction CPL 2.106). This compliance directive provides for uniform procedures and guidelines to be followed when conducting inspections and issuing citations in the absence of a TB standard.

The compliance directive focuses on the primary control measures that are outlined in the CDC guidelines: early 
identification, healthcare-worker screening and follow-up after exposure, and engineering controls, eg, ventilation in isolation and treatment rooms. In addition, the directive provides a summary of the new requirements for respiratory protection that include the use of N95 respirators certified under the revised NIOSH certification procedures and requirements for policies for reuse of disposable respirators. Most of the directive reflects recommendations from the $\mathrm{CDC}$. There are a few components that go beyond the $\mathrm{CDC}$ requirements, such as the requirement for two-step PPD baseline skin testing on all employees. Also, there is a requirement for isolation room signage to include (1) the need for a warning sign (eg, "STOP," "HALT," or biological hazard symbol); (2) isolation sign (eg, “AFB" or "respiratory isolation"); and, (3) a description of the necessary precautions to use (eg, don respirators before entering).

Inspections will be conducted in response to employee complaints, fatalities, or as part of a routine industrial hygiene inspection in workplaces that have been identified as having workers with a greater risk of TB than the general population. These include healthcare facilities, correctional institutions, long-term-care facilities, homeless shelters, and drug treatment centers. OSHA states that healthcare facilities include hospitals where patients with confirmed or suspected TB are treated or to which they are transported. Coverage of nonhospital healthcare settings (ie, doctors' offices, clinics, etc) include only personnel present during the performance of high-hazard procedures on suspect or active TB patients. Dental healthcare personnel are covered by this directive only if they treat suspect or active TB patients in a hospital or correctional facility.

This directive provides OSHA's enforcement procedures for TB until a final TB standard is complete. It is expected that the proposed TB standard will be published in the Federal Register for public comment by the end of this year. Copies of the document are available on the Internet at http://www.osha-slc.gov.

FROM: US Department of Labor: OSHA Office of Health Compliance. Enforcement Procedures and Scheduling for Occupational Exposure to Tuberculosis. (OSHA Instruction CPL 2.106) February 9, 1996.

\section{New York City Strain of MDR-TB Spreads to Four States}

Pablo Bifani of the TB Center of the New York Public Health Research Institute and colleagues recently conducted a study to determine whether isolates of Mycobacterium tuberculosis from New York City and elsewhere that are resistant to four or more primary antimicrobial agents and responsible for widespread disease in the 1990s represent a newly emerged clone or a heterogeneous array of unrelated organisms.

Multidrug-resistant tuberculosis (MDR-TB) isolates were recovered from 253 New York City patients and had the same or closely related fingerprint and hybridization patterns, polymerase chain reaction profiles, and DNA gene sequencing containing mutations associated with resistance to rifampin, isoniazid, and streptomycin. These strains were arbitrarily designated as "W" strains. MDR-TB isolates with these same molecular characteristics were recovered from patients in Florida and Nevada, healthcare workers in Atlanta, Georgia, and Miami, Florida, and an individual who had moved from New York City to Denver, Colorado; and caused disease or skin-test conversion in at least 12 people in a nursing home environment.

The authors conclude that their findings document the molecular origin and spread of a closely related family of MDR-TB strains that have shared a common ancestor and undergone clonal expansion. Dissemination of these difficult-to-treat bacteria throughout New York City, and to at least four additional US cities, has adverse implications for TB control in the 21st century.

Although there has been a $21 \%$ decrease in the number of TB cases reported in New York City between 1992 and 1994, several factors suggest that the long-term ability to eradicate these multidrug-resistant strains will be difficult. First, one study documented 32 healthcare workers (HCWs) who became skin-test positive during an outbreak caused by a W strain. If all HCWs are immunocompetent, $5 \%$ of these individuals will develop active TB in their lifetimes. Second, the strain has spread to at least three major US cities (and four states) that have a combined population of 7.1 million and are hubs for air and auto travel. Third, there are no new primary anti-TB medications introduced since the 1960 s, and it is unlikely that there will be any new drugs. Moreover, even successful, directly observed therapy programs are compromised with MDR-TB.

FROM: Bifani PJ, Plikaytis BB, Kapur V, et al. Origin and interstate spread of a New York City MDR-TB clone family. JAMA 1996;275:452-457.

\section{FDA's Final Medical Device Reporting Regulations}

FDA has issued the final Medical Device Reporting regulation for reporting adverse events to manufacturers. It will be effective on April 11, 1996. This regulation is the last component of the Safe Medical Device Act of 1990, giving the FDA the authority to require manufacturers, distributors, and device-user facilities to submit reports to the FDA on certain types of medical-device-related adverse events.

The Medical Device Reporting regulation provides mechanisms for FDA and manufacturers to identify and monitor significant adverse events involving medical devices, so that problems may be detected and corrected in a timely manner. Under this regulation, medical facilities (referred to as device-user facilities) are required to report to the device manufacturer when a device has or may have caused or contributed to a patient death or serious injury. In the case of death or serious injury, user facilities also must send a copy of the report to the FDA.

In addition to individual device reports, medical facilities also must submit semiannual reports to the FDA, establish files related to reportable events, and maintain those files for 2 years. Further, medical facilities must 